\title{
Presyncope, CTCAE
}

National Cancer Institute

\section{Source}

National Cancer Institute. Presyncope, CT CAE. NCI Thesaurus. Code C143778.

A disorder characterized by an episode of lightheadedness and dizziness which may precede an episode of syncope. 